\title{
Research on the Dynamic Vibration Control of Underwater Robot
}

\author{
Li-ya Wang ${ }^{1}$ and Yang Zhao ${ }^{2, *}$ \\ ${ }^{1}$ School of Mathematics and Information Science, Langfang Teachers University, Langfang 065000, China \\ ${ }^{2}$ Department of Electronic and Information Technology, Jiangmen Polytechnic, Jiangmen 529090, China
}

\begin{abstract}
With mankind's increasing development and utilization of the ocean, underwater robot which can detect underwater environment and autonomously accomplish specific tasks has drawn great attention of researchers. Research was conducted on the kinematic and dynamical control of the underwater robot with six degrees of freedom. Firstly, considering the influences of gravity, buoyancy force, thrust and hydrodynamic force, the kinematic model and dynamical model of the underwater robot were built to describe the complicated underwater behaviors of the robot. Based on this, the dynamic model was simplified and model prediction control of the dynamics was conducted. Comprehensive comparison was also conducted on the results, which showed that model prediction control can reduce the vibration response of the system effectively.
\end{abstract}

Keywords: Dynamics, kinematics, model prediction control, underwater robot.

\section{INTRODUCTION}

Ocean reserves abundant resources and energy sources. How to reasonably use and develop the ocean has become an urgent problem and is drawing wide attention of the all countries. Underwater robot plays an important role in the ocean utilization and development process. With the acceleration of ocean development process, researches on the underwater robots which can explore unknown underwater environment and perform specific underwater tasks also draw the wide attention of research institutions. As the carrier for working in the complicated ocean environment, autonomy and security are the important features of underwater robots, and intelligent control technology is the important foundation and technology for ensuring its autonomy and security $[1,2]$. To cater to the demands of ocean development such as marine oil exploitation etc., the development and application work for underwater robot have been actively conducted in the recent years. Research institutions such as Shanghai Jiaotong University have developed remotely operated vehicle, undersea vehicles and autonomous underwater vehicle etc. W.Naeem et al. [3, 4] conducted on linear model identification for the heading motion of

Hammer head underwater robot. They changed the heading of the underwater robot by controlling the deviation of the rudder, and built ARX model. They conducted on model prediction control for QinetiQ underwater robot with ARX model as the prediction model. K.Ishii et al. [5, 6] built a dynamic model for the experimental platform of TWINBUEGER underwater robot by regressing from the output layer to the input layer of the neural network based on Elman neural network. The dynamic model of the neural network

*Address correspondence to this author at the School of Department of Electronic and Information Technology, Jiangmen Polytechnic, Jiangmen, 529090, China; Email: zhaoyang19781023@gmail.com for this underwater robot is mainly used for on-line adjusting the parameters of the motion controller of the underwater robot. F.J.Song [7] controlled the pitch and degree of freedom direction of OEX underwater robot, and proved the effectiveness of fuzzy sliding mode controller. However, the vibration of the mechanical system in the motion process reduces the motion precision of the mechanism or makes the mechanism deviate from the expected motion. To reduce the vibration characteristics of the mechanism, it needs to compensate the vibration error by a reasonable control system, so as to enable the mechanism to realize the maximum expected motion track.

\section{KINEMATIC AND DYNAMIC EQUATION ANAL- YSIS}

\subsection{Kinematic Equation Analysis}

Underwater robot is shown in Fig. (1). To describe the motion of the underwater robot, the kinematic model of the underwater robot needs to be built according to its motion tracks and motion speeds planned for different tasks. In the fixed coordinate system, the position and posture of the underwater robot can be shown by the coordinates of the underwater robot's gravity in the fixed coordinate system $(x, y, z)$ and Eulerian angles $(\alpha, \beta, \gamma)$ between the moving coordinate system and the fixed coordinate system. The instantaneous linear velocity and angular velocity of the underwater robot are $(p, q, r)$ and $(\mu, v, \omega)$ respectively, and components of the forces and moments applied on each axis of the underwater robot are $(X, Y, Z)$ and $(K, M, Z)$ respectively. The direction of the velocity and the force is consistent with the coordinate axis, and the angular velocity and moment direction are judged according to right-hand screw rule.

The pathway and velocity of the underwater robot planned in the fixed coordinate system needs to be converted 


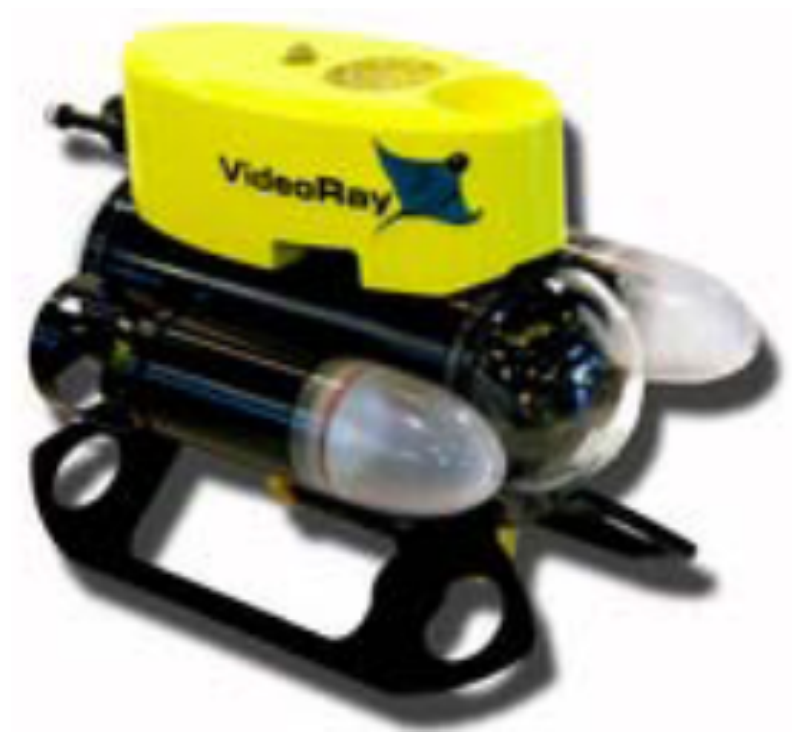

Fig. (1). Underwater robot and the kinematic diagram of its mechanism.

to the moving coordinate system. The velocity generated by the force (moment) applied on the underwater robot needs to be converted to the fixed coordinate system. Only by doing so can make the current position and posture of the robot be calculated. Therefore, the vector conversion between the two coordinate systems is necessary.

For linear velocity, conversion matrix $\mathbf{J}_{v}$ from the moving coordinate system to the fixed coordinate system is as follows:

$$
\mathbf{J}_{v}=\left[\begin{array}{ccc}
c \beta c \gamma & s \alpha s \beta c \gamma-c \alpha s \gamma & c \alpha s \beta c \gamma+s \alpha s \gamma \\
c \theta s \gamma & s \alpha s \beta s \gamma+c \alpha c \gamma & c \alpha s \beta s \gamma-s \alpha c \gamma \\
-s \beta & s \alpha c \beta & c \alpha c \beta
\end{array}\right]
$$

For angular velocity, the conversion matrix $\mathbf{J}_{w}$ from the moving coordinate system to the fixed coordinate system is as follows:

$$
\mathbf{J}_{w}=\left[\begin{array}{ccc}
1 & \frac{s \alpha s \beta}{c \beta} & \frac{c \alpha s \beta}{c \beta} \\
0 & c \alpha & -s \alpha \\
0 & \frac{s \alpha}{c \beta} & \frac{c \alpha}{c \beta}
\end{array}\right]
$$

The conversion from the fixed coordinate system to the moving coordinate system is as follows:

$$
\left[\begin{array}{l}
v_{1} \\
v_{2}
\end{array}\right]=\left[\begin{array}{cc}
\mathbf{J}_{v}^{T} & \mathbf{O} \\
\mathbf{O} & \mathbf{J}_{w}^{-1}
\end{array}\right]\left[\begin{array}{l}
\dot{\eta}_{1} \\
\dot{\eta}_{2}
\end{array}\right]
$$

Where

$$
\begin{aligned}
\eta_{1}^{T} & =[x, y, z], \eta_{2}^{T}=[\alpha, \beta, \gamma], \\
v_{1}^{T} & =\left[\begin{array}{lll}
p & q & r
\end{array}\right], v_{2}^{T}=\left[\begin{array}{lll}
u & v & w
\end{array}\right]
\end{aligned}
$$

\subsection{Dynamic Equation Analysis}

According to Newton-Euler equations for rigid body in fluid, the dynamic model of the underwater robot with six degrees of freedom in the moving coordinate system can be described as follows[8]:

$$
\begin{aligned}
& M \dot{v}+D(v) v+g(\eta)=\tau \\
& \dot{\eta}=\mathbf{J}(\eta) v
\end{aligned}
$$

\section{MODEL SIMPLIFICATION AND CONTROL MODEL BUILDING}

The origin point of the coordinate system is consistent with the center of gravity for the underwater robot, and the buoyancy force equals to the gravity. Only the elements on the diagonal line between the mass inertia matrix and the damping matrix of the hydrodynamic force are considered, the rolling and tumbling motions are passive and controllable, i.e., they can be neglected. The pitching and rolling motions are controlled by the righting moment generated by the gravity and the buoyancy force to minimize the pitching and rolling angles. The underwater robot is symmetrical about three tangent planes. The motion of underwater robot in sideway degree of freedom direction is often neglected. Coriolis and centripetal force can be neglected under low speed. Therefore, the dynamic model of the underwater robot can be further simplified as follows:

$M \dot{v}+D(v) v+g(\eta)=\tau$

Simplification of the mass and inertia matrix as follows: the origin point of the moving coordinate system is located at the center of gravity for the underwater robot, and the underwater robot is symmetrical about three tangent planes. Simplification for the vector of gravity and buoyancy force as follows: in the moving coordinate system, the coordinate of the center of gravity for the underwater robot is $r_{G}=\left[\begin{array}{lll}0 & 0 & 0\end{array}\right]^{T}$, and the coordinate of its buoyancy force is $r_{B}=\left[\begin{array}{lll}0 & 0 & -0.1\end{array}\right]^{T}$. Therefore, the buoyant center is also on 

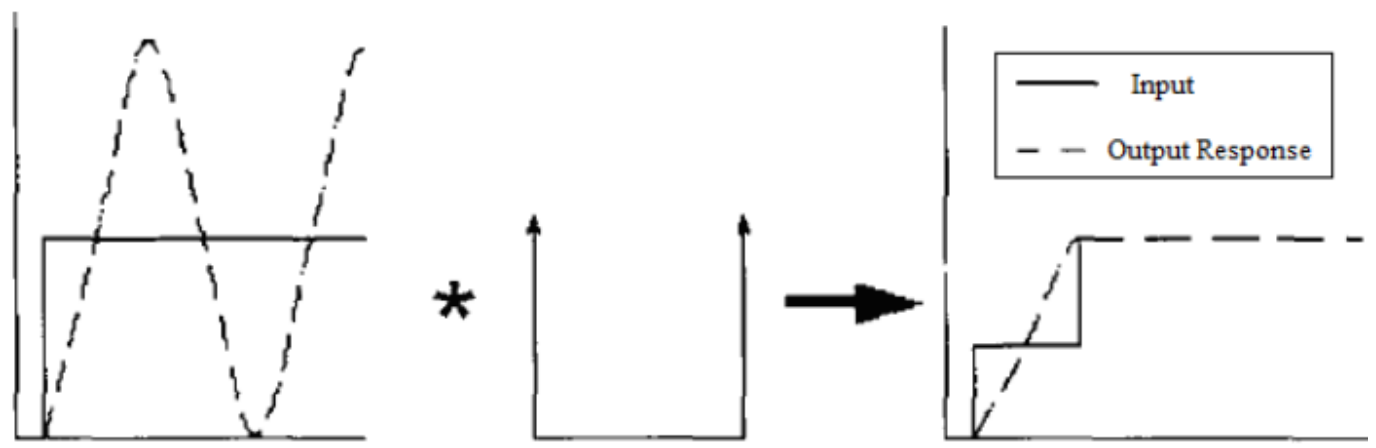

Fig. (2). Diagram of input shaping.

$O Z$ coordinate axis. The gravity of the underwater robot is $1166 \mathrm{~N}$, and the buoyancy force of the water applied on the underwater robot is $1182 \mathrm{~N}$. Therefore, the resultant force of the gravity and the buoyancy force applied on the underwater robot will only affect its motion in heaving degree of freedom direction. Moreover, when the underwater robots is immersed in the water completely, the buoyancy force of the water applied on the underwater robot is $16 \mathrm{~N}$ larger than its gravity, which can ensure that the underwater robot can float upwards by relying on its buoyancy force in case of failure.

\section{RESEARCH ON THE METHODS OF ACTIVE CONTROL}

The elastic deformation of the components will cause dynamic error for its output motion; therefore, it cannot accurately realize the expected track. The dynamic error of the underwater robot is mainly caused by the vibration of the components during the vibration of the system. Vibration will distort the motion track of the system and deviates the input and output relation. How to effectively control the vibration of the mechanism to increase the motion precision of the system and reduce the industrial noise caused by vibration is of great practical significance [9]. For active vibration control, model reduction is conducted by modal truncation during controller design. Neglecting high-frequency dynamic problems may cause failure to observation and control, and there is external perturbation and uncertainties to the actual system, so it needs to increase the robustness of the control system.

\subsection{Dynamic Vibration Control Based on PZT Intelligent Control Material}

Vibrations will occur during the motion of robots. The vibrations not only cause noise, but also affect the motion precision. So it needs to reduce the vibrations. There are many factors causing system vibration. Therefore, it needs a real-time vibration control method to reduce the vibration.

In recent several decades, attention has been paid to retraining the vibration by intelligent structure. An intelligent structure includes four factors: actuator, sensor, control strategy and dynamic control device. Piezoelectric materials can be used as the actuator and sensor of an intelligent structure. PZT intelligent piezoelectric material requires lower driving voltage and can be applied in larger frequency range. Therefore, it is widely used [10]. PZT sensor and actuator coordi- nated with multiple control strategies can realize dynamic vibration control for robots, of which strain rate feedback control strategy has wider dynamic damping frequency area, and can realize dynamic restrain in larger area.

Because of piezoelectric characteristics, PZT intelligent material can be used as a sensor to measure the vibration and as an actuator to restrain the vibration. The control methods for restraining vibration are model prediction control.

\subsection{Theory and Methods of Input Shaping}

Input shaping is to convolve the input signals and a series of pulses, and then input the convolved results to the actuator, thus reducing the vibration of the system. The amplitude and work time of the pulses can be calculated by the resonant frequency and damping ratio of the system. When the last pulse work ends, the vibration of the system shall be the minimum. As shown in Fig. (2), it is the execution process of the convolution between a two-pulse input shaper and the input signals.

\subsection{ZVD Input Shaper}

When the accurate resonant frequency and damping ratio of the system is obtained in advance, ZV input shaper will be able to reduce the residual vibration of the system. However, in case that there are some errors or fluctuation in building model, the resonant frequency and damping ratio of the system, ZV input shaper will not be able to effectively restrain the residual vibration. So the robustness of the input shaper must be improved.

In case of dynamic parameter change or error in the system, Zero Vibration and Derivative (ZVD) method will become a method which can effectively improve the robustness of the shaper. The residual vibration of the system can be indicated by $V(\omega, \zeta)$.

$$
\begin{aligned}
& V(\omega, \zeta)=\mathrm{e}^{-\zeta \omega t_{n}}\left(\sum_{i=1}^{n}\left[\mathrm{~A}_{i} \mathrm{e}^{-\zeta \omega\left(\mathrm{t}-\mathrm{t}_{i}\right)} \cos \left(\omega \sqrt{1-\zeta^{2}} \mathrm{t}_{i}\right)\right]^{2}+\right. \\
& \left.\sum_{i=1}^{n}\left[\mathrm{~A}_{i} \mathrm{e}^{-\zeta \omega\left(\mathrm{t}-\mathrm{t}_{i}\right)} \sin \left(\omega \sqrt{1-\zeta^{2}} \mathrm{t}_{i}\right)\right]^{2}\right)^{\frac{1}{2}}
\end{aligned}
$$

To improve the robustness of the shaper, a constraint equation is added as follows.

$\frac{\partial V(\omega, \zeta)}{\partial \omega}=0$ 


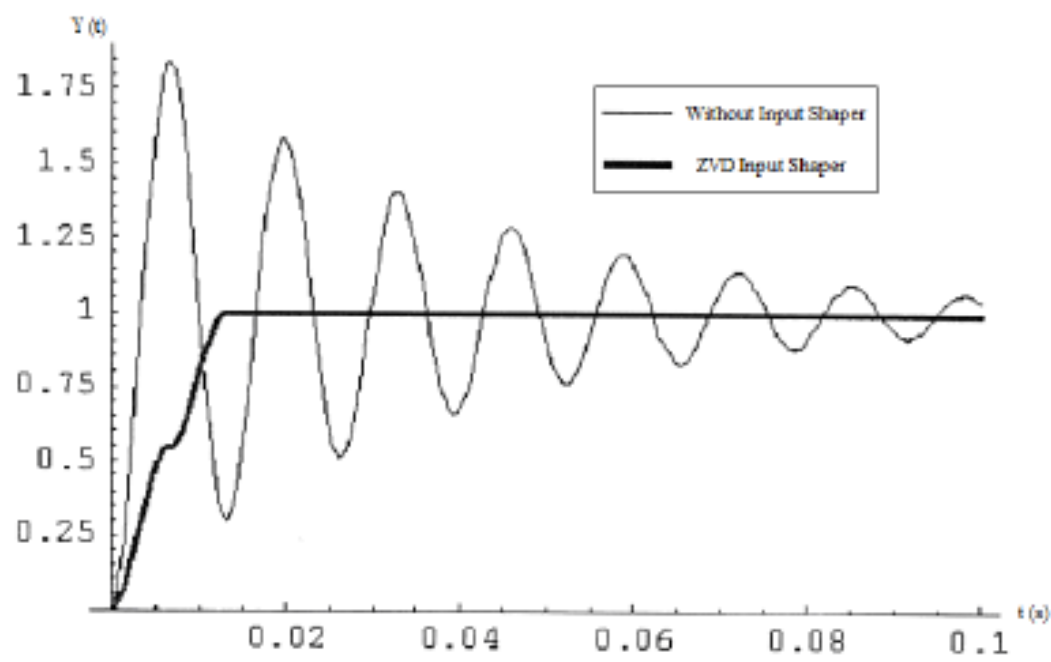

Fig. (3). Comparison of the vibrations without input shaping and with ZVD input shaping.

This constraint equation shows that the derivative of constraint equation of residual vibration to the frequency is made to be zero. All the constraint equations of ZVD method are as follows:

$$
V(\omega, \zeta)=0, \sum_{i=1}^{n} A_{i}=1, \frac{\partial V(\omega, \zeta)}{\partial \omega}=0
$$

Where $V(\omega, \zeta)=0$ is to ensure that the vibration amplitude of the residual vibration is zero when the last pulse work ends. ZVD input shaper not only requires to limit the vibration modal of the system, but also requires that the change rate of the system vibration equals to zero, so make $\dot{V}(\omega, \zeta)=0$ means that it requires zero derivative.

\subsection{Simulation Analysis}

To enhance the robustness of the input shaper, the reduction vibration effect of ZVD input shaping method can be analyzed by simulation. The first order damping ratio and resonant frequency are $\zeta=0.057$ and $f=76.6 \mathrm{~Hz}$ respectively. The input signals of the system are unit step signals.

The parameters of the three-pulse ZVD input shaper for the robot are as follows:

$$
\left[\begin{array}{l}
A_{i} \\
t_{i}
\end{array}\right]=\left[\begin{array}{ccc}
0.2967 & 0.496 & 0.2072 \\
0 & 0.0065 & 0.013
\end{array}\right]
$$

The simulation analysis is conducted and the simulation results are shown in Fig. (3), showing the vibration responses of the connecting equipments for the robot with or without ZVD input shaper. The vibration is restrained by $97 \%$, and the delay time of its response is $0.0074 \mathrm{~s}$.

It is difficult to accurately build a systematic mathematical model due to influences of the non-linear factors and the coupling among various factors, which bring difficulty in the active vibration control of the elastic mechanism. Model prediction control method which has flexible forms and requires less for the system model can compensate the control errors caused by the time variation of the system parameters and non-linear factors, etc. in a real-time manner, and can effectively overcome the influences of the uncertainties of the system, with good control effect and strong robustness [10]. The control structure diagram is shown in Fig. (4).

In this section, its space model in discrete state is built. The vibration response of the mechanism is restricted by means of model prediction control. Its schematic diagram is shown in Fig. (4). The prediction model for predicting the dynamic deformation response of the mechanism was built by making the influences of non-linear factors, coupling factors as well as the high order mode of the system in the dynamic model of the complicated mechanism as the perturbation. The prediction model for the dynamic response of the system was built by regarding the modal force as uncertain perturbation and by taking into account the influence of the output noise on the system. The state variables of the system were estimated by Kalman filtering estimator. The controller was obtained by solving quadratic programming optimization problem.

\section{SIMULATION ANALYSIS ON NUMERICAL CAL- CULATION}

With underwater robot as the research object, its controller was designed by applying $H_{\infty}$ norm and $u$ comprehensive robustness control theory. According to the structure characteristics of the transmission motion, the harmonic gear is featured by the flexible joints; the output end of the harmonic reducer is connected to the piezoelectric intelligent flexible beam. And the partial connecting equipments are the applied the ZVD input shaper to reduce the vibration response effectively. This platform is a rigid-flexible coupling system platform integrating sensor, piezoelectric intelligent material sensor and actuator as well as harmonic gear reducer driven by AC servo motor. Comparative study was conducted on the active vibration control methods. The geometric dimension of the flexible link is as follows: length is $0.65 \mathrm{~m}$, width is $0.1 \mathrm{~m}$, thickness is $1.78 \mathrm{~mm}$, structure damping ratio is $0.3 \%$. The mechanical property of the beam is as follows: elasticity modulus $\mathrm{E}=34.64 \mathrm{Gpa}$, Poisson's ratio $\mathrm{u}=0.33$, density $\rho=1840 \mathrm{~kg} / \mathrm{m}^{3}$. The discretely distributed piezoelectric transducer (PZT) sensors are pasted at the root 


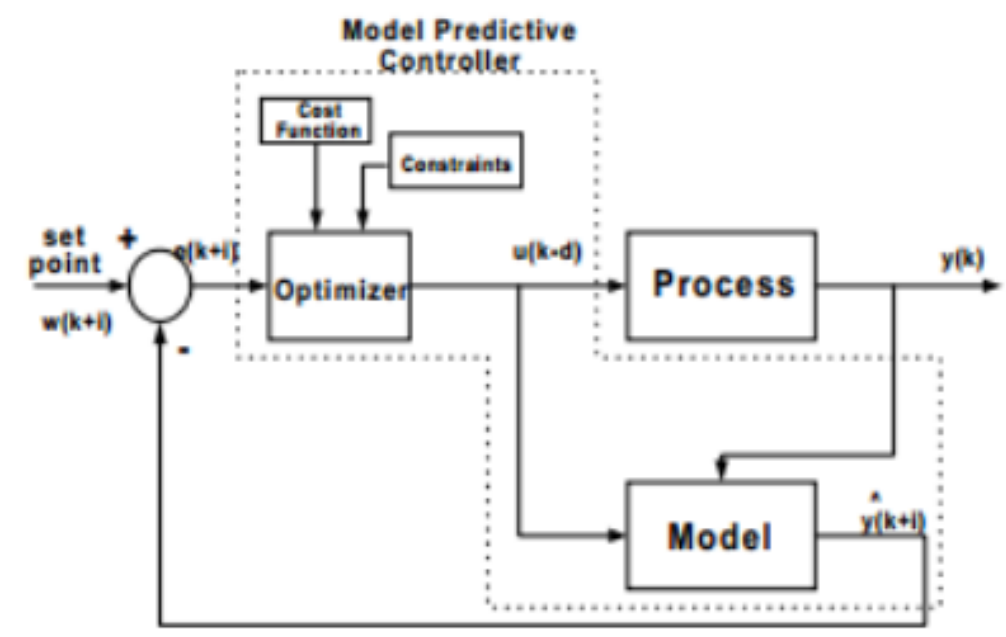

Fig. (4). Schematic diagram of model prediction control.

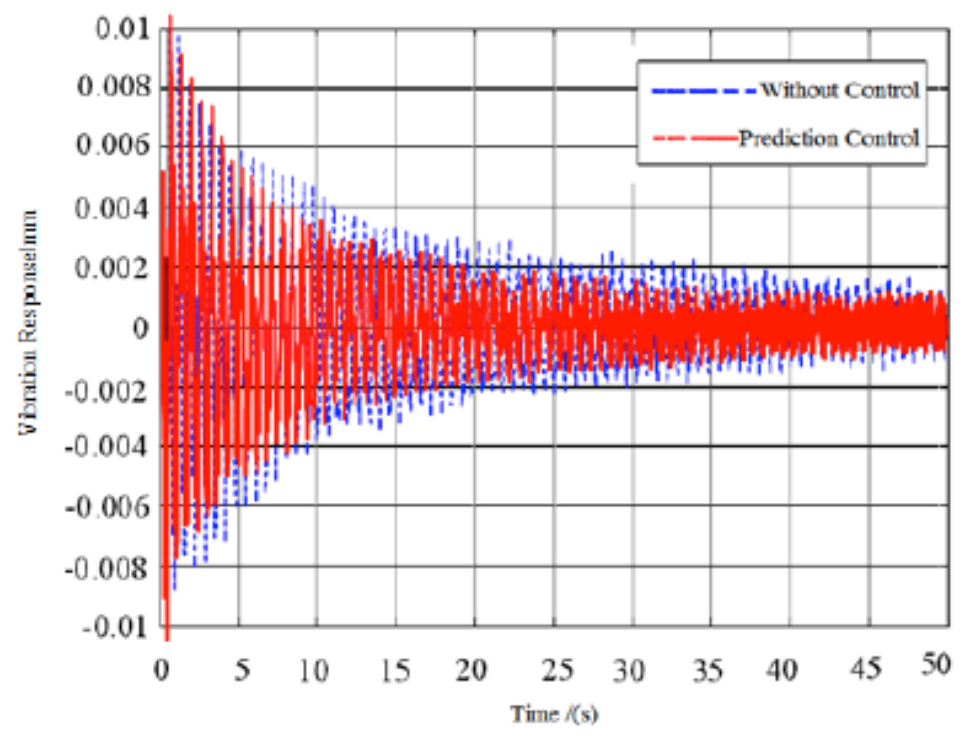

Fig. (5). Simulation and experiment results of model prediction control.

and the middle of the beam. The geometric dimension of the piezoelectric transducer (PZT) is $50 \mathrm{~mm} \times 15 \mathrm{~mm} \times 1 \mathrm{~mm}$, its elasticity modulus, Poisson's ratio, density and piezoelectric strain constant are

$$
E_{p}=6.5 \times 10^{10} \mathrm{pa}, \mu_{p}=0.35, \rho_{p}=7650 \mathrm{~kg} / \mathrm{m}^{3}
$$

and $d=166 \times 10^{-12} \mathrm{~m} / V$ respectively. To verify the effectiveness of the controller, numerical simulation analysis was conducted [11].

The solid line in Fig.5 is prediction control, and the dotted line is the uncontrolled model. Fig. (5) shows that the response of the uncontrolled system is basically consistent with that of the prediction control system. It can well reflect the real characteristics of the uncontrolled model, thus carrying out dynamic characteristic analysis and active control design. Therefore, based on the model of prediction control, the control strategy can be designed according to the optimum control theory, so as to restrain the elastic vibration of the flexible plate.
In conclusion, with the underwater robot with six degrees of freedom as the research object, theoretical and experimental study is conducted on time domain dynamic modeling and active control based on the input and output data of the system. The numerical simulation results show that prediction model control can meet the requirements of active control design, and can effectively reduce the vibration response of the mechanism system. It can reduce the vibration response of the vibration system and reduce the vibration characteristics to the maximum extent, thus improving the motion precision of the mechanism. In conclusion, the control methods proposed in the paper are feasible and effective; the methods based on master control can effectively restrain the elastic vibration of the system.

\section{CONCLUSION}

The kinematic and dynamic model for the underwater robot with six degrees of freedom is built. Based on this, prediction model simulation control was conducted. The simula- 
tion results of the control strategy show that the control strategy proposed in this paper can enable the underwater robot to have strong anti-interference ability and accurately trace the time-varying theoretical trace. Model prediction control can reduce the vibration response of the mechanism to the maximum extent, thus increasing the motion precision of the mechanical system.

\section{CONFLICT OF INTEREST}

The authors confirm that this article content has no conflict of interest.

\section{ACKNOWLEDGEMENTS}

The work was supported by the Langfang Science and Technology Research and development Guidance Program 2014 (2014023095).

\section{REFERENCES}

N. Sakaqami, M. Inoue, and S. Kawamura, "Theoretical and experimental studies on iterative learning control for underwater robots," International Journal of Offshore and Polar Engineering, vol. 13, no. 2, pp. 120-127, 2003.

[2] J. Yuh, "Design and control of autonomous underwater robots: a survey," Autonomous Robots, vol. 8, no. 1, pp. 7-24, 2000.
[3] W. Naeem, R. Sutton, and J. Chudley, "System identification, modeling and control of an autonomous underwater vehicle," In: Proceedings of MCMC 2003 Conference, Girona, Spain, 2003, pp. 37-42.

[4] W. Naeem, "Model predictive control of an autonomous underwater vehicle," In: Proceedings of UKACC 2002 Postgraduate Symposium, Sheffield, UK0002, 2002, pp. 19-23.

[5] K. Ishii, T. Um and T. Vujii, "A feed forward neural network for identification and adaptive control of autonomous underwater vehicles," In: IEEE ICNN, 1994, pp. 3216-3221.

[6] K. Ishii, T. Fujii and T. Ura, "An adaptive neural-net controller system for an underwater vehicle," Control Engineering Practice vol. 8, pp. 177-184, 2000.

[7] R. Cristi, M. Caccia, and G. Veruggio, "Motion estimation and modeling of the environment for underwater vehicle," International Journal of Systems Science, vol. 29, no. 10, pp. 1135-1143, 1998.

[8] C. Puaut, "Hydrodynamic Analysis of Autonomous Underwater Vehicles in Shallow Water Waves," MS Thesis, Department of Ocean Engineering, Flordia Atlantic University, 2001.

[9] D. Cortes, N. Vazquez, and J. A. Gallegos, "Dynamical slidingmode control of the boost inverter," IEEE Transactions on Industrial Electronics, vol. 56, no. 9, pp. 3467-3476, 2009.

[10] S. Liu, L. Tong, and Z. Lin, "Simultaneous optimization of control parameters and configuration of PZT actuators for morphing structural shapes," Finite Elements in Analysis and Design, vol. 44, pp. 417-424, 2008.

[11] K.Y. Pettersen, and H. Nijmeijer, "Under actuated Ship Tracking Control: Theory and Experiments," International Journal of Control, vol. 74, no. 14, pp. 1435-1146, 2001

(C) Wang and Zhao; Licensee Bentham Open.

This is an open access articles licensed under the terms of the Creative Commons Attribution-Non-Commercial 4.0 International Public License (CC BY-NC 4.0) (https://creativecommons.org/licenses/by-nc/4.0/legalcode), which permits unrestricted, non-commercial use, distribution and reproduction in any medium, provided that the work is properly cited. 